\title{
Eficiência Biológica de Vacas de Dois Grupos Genéticos Amamentando Bezerros Puros ou F1, Mantidas em Diferentes Condições de Alimentação
}

\author{
João Restle ${ }^{1}$, Paulo Santana Pacheco ${ }^{2}$, João Teodoro Pádua ${ }^{3}$, Marta Gomes da Rocha ${ }^{4}$, Ricardo \\ Zambarda Vaz ${ }^{5}$, Eduardo da Costa Eifert ${ }^{6}$, José Luis Moletta ${ }^{7}$, Aline Kellermann de Freitas ${ }^{8}$
}

RESUMO - O experimento foi conduzido com o objetivo de avaliar a eficiência biológica de vacas dos grupos genéticos Charolês $(\mathrm{CH})$ e Nelore (NE) amamentando bezerros $\mathrm{CH}$ e $1 / 2 \mathrm{NE} 1 \frac{1}{2} \mathrm{CH}$ (filhos de vacas $\mathrm{CH}$ ), $\mathrm{NE}$ e $1 / 2 \mathrm{CH} 1 / 2 \mathrm{NE}$ (filhos de vacas $\mathrm{NE}$ ) mantidos juntos até o desmame, em pastagem nativa (PN) ou pastagem cultivada (PC) de inverno (aveia + azevém) e de verão (capim papuã). Vacas e bezerros foram pesados ao nascimento, aos 14, 42, 70, 98, 126, 154 e 182 dias e ao desmame. A produção de leite das vacas foi avaliada na mesma data das pesagens, com exceção do nascimento. Quando os animais foram mantidos em PC, observou-se maior eficiência na transformação da quantidade de litros (EFLIT) (7,7 contra 11,0 L/kg) e matéria seca (MS) (EFMS) de leite (1,06 contra 1,47 kg MS/kg) produzido pela vaca em $\mathrm{kg}$ de ganho de peso do bezerro. Foi verificado efeito quadrático do período de aleitamento para EFLIT ( $\mathrm{y}=18,222-$ 0,141DIA+0,000463 DIA ${ }^{2}$ ) e EFMS ( $\mathrm{y}=2,341-0,0182 \mathrm{DIA}+0,000063 \mathrm{DIA}^{2}$ ). Houve redução linear, com o avanço do período de lactação, na exigência diária de nutrientes digestíveis totais (mantença + produção de leite) (NDT) (NDT/dia) para vacas mantidas em PC $(0,004 \mathrm{~kg})$ e PN $(0,007 \mathrm{~kg}$ ). A exigência total (parto ao desmame) de NDT (NDT/total) foi superior para as vacas mantidas em PC (1093,1 contra 1014,0 $\mathrm{kg})$. Contudo, vacas mantidas em PC foram mais eficientes na transformação de NDT/dia $(7,9$ contra 12,5) e NDT/total $(5,8$ contra 8,2) em ganho de peso do bezerro. A quantidade de $\mathrm{kg}$ de bezerros produzidos $/ 100 \mathrm{~kg}$ de vaca (EFKG) $(0,201 \mathrm{contra} 0,159 \mathrm{~kg} / 100 \mathrm{~kg} / \mathrm{dia})$ e $\mathrm{kg}$ de bezerro produzido por unidade de tamanho metabólico da vaca (UTM) (EFTM) $(0,009$ contra $0,007 \mathrm{~kg} / \mathrm{UTM} /$ dia) foram superiores quando as vacas foram mantidas em PC. Vacas amamentando bezerros F1 exigiram mais NDT/dia (5,0 contra 4,8 kg/dia), mas foram mais eficientes para EFKG (27,1 contra 23,5 kg/100 kg) e EFTM (1,21 contra 1,05 kg/UTM). Nas vacas amamentando bezerros mestiços, a EFKG foi maior nas $\mathrm{CH}$ (28,5 contra 25,6 kg/100 kg). Quando foi considerada a EFTM, observou-se maiores valores nas vacas CH $(1,11 \mathrm{~kg} / \mathrm{UTM})$ em relação às $\mathrm{NE}(0,99 \mathrm{~kg} / \mathrm{UTM})$, quando amamentavam bezerros puros, não havendo diferença quando amamentavam bezerros mestiços.

Palavras-chave: Charolês, cruzamento, heterose, idade da vaca, Nelore, produção de leite

\section{Biologic Efficiency of Cows of Two Genetic Groups Nursing Straigthbred or F1 Calves, Mantained on Different Feeding Conditions}

\begin{abstract}
The objective of the experiment was to evaluate the biologic efficiency of Charolais (CH) and Nellore (NE) cows nursing $\mathrm{CH}$ and $1 / 2 \mathrm{NE} 1 / 2 \mathrm{CH}$ (produced by $\mathrm{CH}$ cows), $\mathrm{NE}$ and $1 / 2 \mathrm{CH} 1 / 2 \mathrm{NE}$ (produced by $\mathrm{NE}$ cows) calves, mantained together until weaning, on native pasture (NP) or winter (oat + italian rye grass) and summer (alexander grass) cultivated pasture (CP). Cows and calves were weighted at birth; $14 ; 42 ; 70 ; 98 ; 126 ; 154 ; 182$ days; and at weaning. The cows milk yield was evaluated at the same date of the calves weighing, with exception at birth. When the animals were mantained on CP, higher efficiency of converting the quantity of litres (EFLIT) $(7.7 \mathrm{vs} 11 \mathrm{~L} / \mathrm{kg})$ and dry matter (DM) (EFMS) of milk (1.06 vs $1.47 \mathrm{~kg} \mathrm{DM} / \mathrm{kg}$ ) produced by cow into $\mathrm{kg}$ of calf weight gain, was observed. Quadratic effect of postpartum period for EFLIT $\left(\mathrm{y}=18.222-.141 \mathrm{DAY}+.000463 \mathrm{DAY}^{2}\right)$ and EFMS $\left(\mathrm{y}=2.341-.0182 \mathrm{DAY}+.000063 \mathrm{DAY}^{2}\right)$ was verified. Linear reduction of the daily requeriment of total digestible nutrients (mantainance plus milk production) (NDT) (NDT/day) was observed, as the lactation period advanced, for cows mantained on CP $(.004 \mathrm{~kg})$ and NP $(.007 \mathrm{~kg})$. The total requeriment (calving to weaning) of NDT (NDT/total) was superior for cows mantained on CP (1093.1 vs $1014 \mathrm{~kg})$. However, cows mantained on CP were more efficient for transformation of NDT/dia (7.9 vs $12.5)$ and NDT/total (5.8 vs 8.2) in calf weight gain. The quantity of kg of calf produced/100 kg of cow (EFKG) (.201 vs .159 kg/100kg/day) and $\mathrm{kg}$ of calf produced per unit of cow metabolic weight (UTM) (EFTM) (.009 vs $.007 \mathrm{~kg} / \mathrm{UTM} / \mathrm{day})$ were higher when the cows were mantained on CP. Cows nursing F1 calves required more NDT/day (5 vs $4.8 \mathrm{~kg} /$ day). However, were more efficient for EFKG $(27.1 \mathrm{vs} 23.5 \mathrm{~kg} / 100 \mathrm{~kg}$ ) and EFTM (1.21 vs $1.05 \mathrm{~kg} / \mathrm{UTM}$ ). For cows nursing crossbred calves, the EFKG was higher for CH (28.5 vs $25.6 \mathrm{~kg} / 100 \mathrm{~kg})$. When EFTM was considered, higher value was observed for CH $(1.11 \mathrm{~kg} / \mathrm{UTM})$ than for NE $(.99 \mathrm{~kg} / \mathrm{UTM})$ cows, when they nursed straigthbred calves.
\end{abstract}

Key Words: Charolais, cow age, crossbred, heterosis, milk yield, Nellore

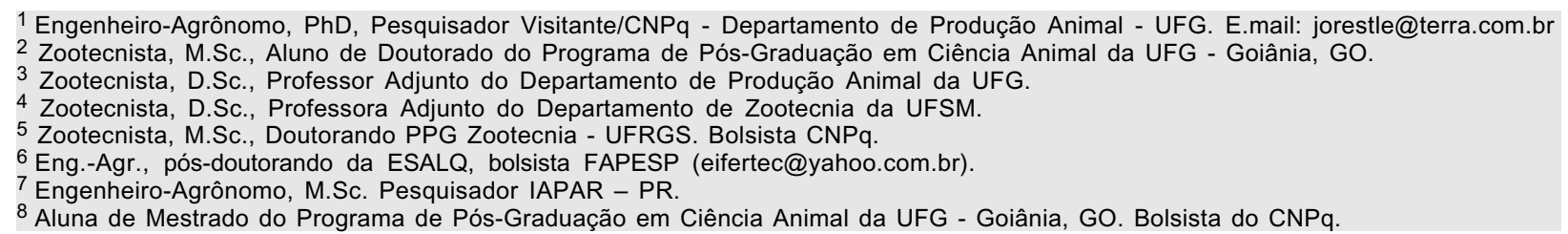




\section{Introdução}

Apesar de o Brasil possuir o maior rebanho comercial do mundo, com aproximadamente 166,8 milhões de cabeças, os índices zootécnicos apresentaram pouco progresso na última década (ANUALPEC, 2002), indicando que a produtividade e a rentabilidade do setor pecuário estão aquém do seu potencial.

Com a atual necessidade da busca pela eficiência produtiva, demandada pela exigência de maior rentabilidade pela pecuária de corte, o produtor deve buscar conhecimento e tecnologias adequadas para serem aplicadas em seu sistema de produção. Em propriedades que utilizam o sistema de ciclo completo, Restle et al. (2003) e Restle et al. (2004) citam que uma alternativa para maximizar a rentabilidade seria aumentar a quantidade de $\mathrm{kg}$ de bezerro desmamado por vaca/ano, o que é determinado pela taxa de desmame e pelo peso médio dos bezerros.

O peso ao desmame é uma característica importante, pois está associado à idade a puberdade nas fêmeas (Restle et al., 1999a) e à idade de abate nos machos (Restle et al., 1999b), sendo influenciado pela habilidade materna da vaca, pelo potencial genético para ganho de peso do bezerro, sexo do bezerro e nível nutricional (Ribeiro \& Restle, 1991; Moojen et al., 1994; Restle et al., 2004).

Vários estudos demonstraram a elevada correlação entre a produção de leite da vaca e o desempenho do bezerro (Melton et al., 1967; Totusek et al., 1973; Ribeiro \& Restle, 1991; Alencar et al., 1996; Restle et al., 2004), sendo a produção de leite influenciada pelos fatores ambiental e genético. Quanto ao fator ambiental, vacas sob melhores condições alimentares produzem mais leite e, conseqüentemente, bezerros mais pesados ao desmame (Neville Jr., 1962; Bond \& Wiltbank, 1970; Restle et al., 2003). Entre os fatores genéticos, destacam-se a composição genética dos animais, expressa pelo grau de sangue ou grupo genético, e o tamanho da vaca, uma vez que vacas maiores dentro do mesmo rebanho ou entre raças apresentam maior produção de leite (Melton et al., 1967; Alencar et al., 1993; Cruz et al., 1997).

Porém, poucos são os estudos sobre a avaliação da eficiência biológica do sistema de cria, persistindo dúvidas do quanto elevar os índices de produção de leite mantém eficiente o processo de produção de $\mathrm{kg}$ de bezerros desmamados. Isto porque a produção de leite exerce efeito negativo nos índices reprodutivos do rebanho (Euclides Filho et al., 1983; Kress et al., 1990;
Senna, 1996; Lobato et al., 1998), além de aumentar os requerimentos nutricionais das vacas que compõem o rebanho de cria (Ferrel \& Jenkins, 1984; Montaño Bermudez et al., 1990; Freking \& Marshall, 1992; Ribeiro et al., 2001).

Resultados de trabalhos comparando a eficiência produtiva de vacas de diferentes genótipos demonstraram que as mais pesadas, que produzem mais leite e desmamam bezerros mais pesados, podem ser menos produtivas (Kress et al., 1990; Ribeiro et al., 2001) ou mais produtivas (Montaño Bermudez et al., 1990; Barcellos et al., 1996) em relação às vacas mais leves.

Quanto à eficiência de conversão do leite materno em ganho de peso do bezerro, Melton et al. (1967) e Alencar (1987) verificaram que bezerros mais pesados foram menos eficientes que os mais leves, em decorrência do aumento no requerimento de manutenção e menor consumo de forragem (Wyatt et al., 1977; Boggs et al., 1980).

Objetivou-se, com o presente estudo, avaliar a eficiência produtiva de vacas Charolês $(\mathrm{CH})$ ou Nelore (NE) e bezerros puros ( $\mathrm{CH}$ ou $\mathrm{NE})$ ou mestiços $(1 / 2 \mathrm{CH}$ $1 / 2 \mathrm{NE}$ ou $1 / 2 \mathrm{NE} 1 / 2 \mathrm{CH}$ ), submetidos a dois tipos de pastagem (nativa ou cultivada de inverno e verão) do nascimento ao desmame. Estudou-se também a relação entre as diferentes medidas de eficiência com a produção de leite e com o desempenho dos bezerros.

\section{Material e Métodos}

O experimento foi conduzido no Setor de Bovinocultura de Corte do Departamento de Zootecnia da Universidade Federal de Santa Maria, em Santa Maria, Rio Grande do Sul, na região fisiográfica Depressão Central, que, segundo classificação de Köppen, apresenta clima subtropical úmido (cfa).

Foram utilizadas 60 vacas, primíparas e pluríparas, com idade de três a treze anos, sendo 30 do grupo genético Charolês $(\mathrm{CH})$ e 30 do grupo genético Nelore (NE), tomadas ao acaso do mesmo rebanho experimental. As vacas amamentavam bezerros puros $(\mathrm{CH}$ ou $\mathrm{NE})$ ou mestiços $(1 / 2 \mathrm{CH} 1 / 2 \mathrm{NE}$ ou $1 / 2 \mathrm{NE} 1 / 2 \mathrm{CH})$.

Foram estudados os seguintes tratamentos:

CHPN - Vacas CH mantidas em pastagem nativa (PN), do parto ao desmame, amamentando bezerros puros $(n=10)$ ou mestiços $(n=5)$;

NEPN - Vacas NE mantidas em PN, do parto ao desmame, amamentando bezerros puros $(n=10)$ ou mestiços $(n=5)$; 
CHPC - Vacas CH mantidas em pastagem cultivada (PC) de aveia (Avena sativa) mais azevém (Lollium multiflorum) do parto até o final do mês de novembro e, após, em pastagem de capim papuã (Brachiaria plantagínea) até o desmame, amamentando bezerros puros $(n=7)$ ou mestiços $(n=8)$;

NEPC - Vacas NE mantidas em PC de aveia (Avena sativa) mais azevém (Lollium multiflorum) do parto até o final do mês de novembro e, posteriormente, em pastagem de capim papuã (Brachiaria plantagínea) até o desmame, amamentando bezerros puros $(n=8)$ ou mestiços $(n=7)$;

Nos tratamentos, os animais ficaram equilibrados pela ordem de parição da vaca.

As vacas foram classificadas em três classes de idade: jovens (3-4 anos), adultas (5-7 anos) e velhas ( 8 anos ou mais).

O período de parição foi de setembro a dezembro, sendo os bezerros obtidos de inseminação artificial (período de 45 dias) e de monta natural (período de 45 dias). Durante o período de inseminação, foi utilizado o sêmen de oito touros $\mathrm{CH}$ e $\mathrm{NE}$ e, para o repasse, quatro $\mathrm{CH}$ e quatro NE. Os touros que produziram os bezerros $\mathrm{CH}$ foram os mesmos que produziram os $1 / 2 \mathrm{CH} 1 / 2 \mathrm{NE}$ e aqueles que produziram os bezerros $\mathrm{NE}$ foram os mesmos que produziram os $1 / 2 \mathrm{NE} 1 / 2 \mathrm{CH}$.

Foi utilizada uma área de 17 ha, com pastagem de inverno (aveia + azevém), e outros 17 ha com pastagem de verão (capim papuã), sendo a lotação/ha de 1,76 vacas com bezerro ao pé para ambos os tipos de PC. Para os animais mantidos em PN, foi utilizada uma área de 30 ha, com lotação/ha de 1,0 vaca com bezerro ao pé.

As vacas e os bezerros foram pesados nas primeiras 24 horas pós-parto, aos 14, 42, 70, 98, 126, 154 e 182 dias e ao desmame, que ocorreu próximo aos 217 dias de idade dos bezerros.

Também foram verificadas as produções de leite das vacas, utilizando o método direto, com ordenha manual, seguindo a técnica descrita por Ribeiro et al. (1991). As avaliações foram realizadas aos 14 dias pós-parto e, a partir daí, com intervalos de 28 dias até o desmame dos bezerros. Após a ordenha, foram coletadas amostras de leite, para determinação da percentagem de gordura, de lactose, de extrato seco total e de extrato seco desengordurado do leite.

As medidas de eficiência produtiva foram: litros de leite produzidos pela vaca por $\mathrm{kg}$ de ganho de peso do bezerro (EFLIT); matéria seca de leite produzido pela vaca por $\mathrm{kg}$ de ganho de peso do bezerro (EFMS); quantidade diária (NDT/dia) e total (NDT/ total) de nutrientes digestíveis totais (NDT) $(\mathrm{kg})$ exigidos pela vaca; NDT por $\mathrm{kg}$ de ganho de peso do bezerro (EFNDT); exigência total de NDT pela vaca por $\mathrm{kg}$ de bezerro desmamado (EFDESM); $\mathrm{kg}$ de bezerros desmamados por $100 \mathrm{~kg}$ de vaca (EFKG); $\mathrm{kg}$ de bezerros desmamados por unidade de peso metabólico da vaca (EFTM), em g. A quantidade de NDT foi determinada pelo somatório do requerimento de manutenção e produção de leite da vaca, sendo que, para vacas primíparas, houve acréscimo de $20 \%$ nos requerimentos de manutenção e nas de segunda parição, acréscimo de 10\% (NRC, 1973).

Foi calculada a heterose $(\mathrm{H})$ para as características: EFLIT, EFMS, NDT, EFNDT, EFKG, e EFTM, pela seguinte fórmula: $\mathrm{H}(\%)=$ (média dos $\mathrm{F} 1 /$ média dos puros -1$) * 100$.

O delineamento experimental utilizado foi o inteiramente casualizado, sendo os dados submetidos à análise de variância, ao teste $\mathrm{F}$ e ao teste de comparação de médias de Tukey, considerando significância de $10 \%$, por intermédio do programa estatístico SAS (1997). O modelo estatístico adotado foi:

$$
\begin{gathered}
\mathrm{Y}_{i j k l m n o p}=\mathrm{m}+\mathrm{NT}_{i}+\mathrm{GG}_{j}+\mathrm{P}_{k}+\mathrm{SA}_{p}+\mathrm{RT}_{l} \\
(\mathrm{SA})_{\mathrm{p}}+\left(\mathrm{P}^{*} \mathrm{GG}\right)_{k j}+\mathrm{S}_{m}+\left(\mathrm{GG}^{*} \mathrm{~S}\right)_{j m}+\mathrm{IV}_{n}+ \\
(\mathrm{IV} * \mathrm{~S})_{n m}+\left(\mathrm{IV}^{* \mathrm{P}}\right)_{n k}+\left(\mathrm{GG}^{*} \mathrm{IV}\right)_{j n}+\mathrm{DIA}_{o}+ \\
(\mathrm{P} * \mathrm{DIA})_{k o}+\left(\mathrm{GG}^{*} \mathrm{DIA}\right)_{j o}+\left(\mathrm{P}^{*} \mathrm{GG}^{*} \mathrm{DIA}\right)_{k j o}+ \\
\sum_{i j k l m n o p}
\end{gathered}
$$

em que $\mathrm{Y}_{i j k l m n o p}=$ variáveis dependentes; $\mathrm{m}=$ média geral de todas as observações; $\mathrm{NT}_{i}=$ efeito da covariável "ordem de nascimento do bezerro" de ordem "i"; $\mathrm{GG}_{j}=$ efeito do grupo genético da vaca de ordem “ $\mathrm{j}$ ”, sendo $1=$ Charolês e $2=$ Nelore $; \mathrm{P}_{k}=$ efeito do tipo de pastagem de ordem "k", sendo $1=$ pastagem cultivada e 2 =pastagem nativa; $\mathrm{SA}_{p}=$ efeito do sistema de acasalamento que originou os bezerros de ordem " $\mathrm{p}$ ", sendo $1=$ puros e $2=$ mestiços; $\mathrm{RT}_{l}(\mathrm{SA})_{p}$ = efeito da raça do bezerro de ordem "l" sendo $1=$ Charolês, $2=$ Nelore, $3=1 / 2$ Charolês $1 / 2$ Nelore e $4=1 / 2$ Nelore $1 / 2$ Charolês dentro do sistema de acasalamento de ordem "p"; $(\mathrm{P} * \mathrm{GG})_{k j}=$ interação entre k-ésimo tipo de pastagem e j-ésimo grupo genético da vaca; $\mathrm{S}_{m}=$ efeito do sexo do bezerro de ordem "m", sendo $1=$ macho e $2=$ fêmea; $\left(\mathrm{GG}^{*} \mathrm{~S}\right)_{j m}=$ interação entre j-ésimo grupo genético da vaca e mésimo sexo do bezerro; $\mathrm{IV}_{n}=$ efeito da classe de idade da vaca de ordem "n", sendo $1=$ jovens, $2=$ adultas e 3=velhas; $\left(I V^{*} \mathrm{~S}\right)_{n m}=$ interação entre n-ésima idade da vaca e m-ésimo sexo do bezerro; $\left(\mathrm{IV}^{*} \mathrm{P}\right)_{n k}$ 
= interação entre n-ésima idade da vaca e k-ésimo tipo de pastagem; $(\mathrm{GG} * \mathrm{IV})_{j n}=$ interação entre jésimo grupo genético da vaca e n-ésima idade da vaca; DIA $_{o}=$ efeito do período de lactação de ordem "o"; (P*DIA $)_{k o}=$ interação entre o k-ésimo tipo de pastagem e o-ésimo período de lactação; (GG*DIA) ${ }_{j o}$ $=$ interação entre o j-ésimo grupo genético da vaca $\mathrm{e}$ o-ésimo período de lactação; $(\mathrm{P} * \mathrm{GG} * \mathrm{DIA})_{k j o}=$ interação entre k-ésimo tipo de pastagem, j-ésimo grupo genético da vaca e o-ésimo período de lactação; $\Sigma_{i j k l m n o p}=$ erro aleatório residual, $\operatorname{NID}\left(0, \sigma^{2}\right)$.

Para estimativas das equações de regressão, o efeito de período (dias pós-parto) sobre as variáveis dependentes foi submetido à análise de regressão polinomial. Para as variáveis dependentes NDT/total e EFDESM, o efeito de período pós-parto foi retirado do modelo.

\section{Resultados e Discussão}

Na Tabela 1, encontram-se os valores médios referentes à quantidade de leite produzido pela vaca, em litros (EFLIT) e em matéria seca (EFMS), para promover $1 \mathrm{~kg}$ de ganho de peso no bezerro, de acordo com o tipo de pastagem, grupo genético da vaca e período pós-parto.

Houve efeito do tipo de pastagem $(\mathrm{P}=0,0183)$ sobre a eficiência de transformar litros de leite produzido em ganho de peso. Bezerros mantidos em pastagem cultivada (PC) foram mais eficientes $(7,7 \mathrm{~L} / \mathrm{kg}$ ) em relação aos bezerros mantidos em pastagem nativa $(\mathrm{PN})(11,0 \mathrm{~L} / \mathrm{kg})$, representando diferença de $30 \%$. Comportamento semelhante foi verificado quando a eficiência foi avaliada a partir da conversão de MS de leite produzido pela vaca em ganho de peso do bezerro (EFMS), havendo influência significativa $(\mathrm{P}=0,0391)$ do tipo de pastagem para esta característica. Bezerros mantidos em PC foram 27,9\% mais eficientes em relação aos mantidos em PN (1,06 contra 1,47).

Estes resultados são explicados pelas diferenças ocorridas na produção de leite das vacas e, principalmente, no ganho de peso médio diário dos bezerros. Apesar de ter havido incremento com a melhoria das condições alimentares (PN para PC) para estas características, as diferenças percentuais foram muito

Tabela 1 - Médias para quantidade de leite produzido pela vaca, em litros (EFLIT) e em kg de matéria seca (EFMS), para cada $\mathrm{kg}$ de ganho de peso do bezerro, de acordo com a pastagem, o grupo genético da vaca e o período pós-parto

Table 1 - Means for quantity of milk produced by cow, in litres (EFLIT) and $\mathrm{kg}$ of dry matter (EFMS), for each $\mathrm{kg}$ of calf weight gain, according to pasture, cow genetic group and postpartum period

\begin{tabular}{|c|c|c|c|c|c|c|c|c|c|}
\hline \multirow[t]{3}{*}{$\begin{array}{l}\text { Pastagem } \\
\text { Pasture }\end{array}$} & \multirow{3}{*}{$\begin{array}{c}\text { Grupo genético } \\
\text { da vaca } \\
\text { Cow genetic } \\
\text { group }\end{array}$} & \multicolumn{6}{|c|}{$\begin{array}{l}\text { Período pós-parto (dias) } \\
\text { Postpartum period (days) }\end{array}$} & & \\
\hline & & \multicolumn{2}{|c|}{$0-70$} & \multicolumn{2}{|c|}{$71-154$} & \multicolumn{2}{|c|}{$\begin{array}{l}\text { 155-desmame } \\
\text { 155-weaning }\end{array}$} & \multicolumn{2}{|c|}{$\begin{array}{l}\text { Média } \\
\text { Mean }\end{array}$} \\
\hline & & EFLIT & EFMS & EFLIT & EFMS & EFLIT & EFMS & $\overline{\text { EFLIT }}$ & EFMS \\
\hline Cultivada & Charolês & 8,8 & 1,05 & 6,3 & 0,84 & 7,7 & 1,14 & 7,6 & 1,01 \\
\hline \multirow[t]{2}{*}{ Cultivated } & $\begin{array}{c}\text { Charolais } \\
\text { Nelore } \\
\text { Nellore }\end{array}$ & 8,5 & 1,15 & 7,2 & 1,03 & 8,0 & 1,15 & 7,9 & 1,11 \\
\hline & $\begin{array}{l}\text { Média } \\
\text { Mean }\end{array}$ & 8,6 & 1,10 & 6,7 & 0,94 & 7,8 & 1,14 & $7,7 \mathrm{~B}$ & $1,06 \mathrm{~B}$ \\
\hline Nativa & Charolês & 10,5 & 1,31 & 7,2 & 0,93 & 14,0 & 1,95 & 10,6 & 1,40 \\
\hline \multirow[t]{2}{*}{ Native } & $\begin{array}{c}\text { Charolais } \\
\text { Nelore } \\
\text { Nellore }\end{array}$ & 15,2 & 2,06 & 9,0 & 1,23 & 10,2 & 1,36 & 11,5 & 1,55 \\
\hline & $\begin{array}{l}\text { Média } \\
\text { Mean }\end{array}$ & 12,8 & 1,69 & 8,1 & 1,08 & 12,1 & 1,66 & $11,0 \mathrm{~A}$ & $1,47 \mathrm{~A}$ \\
\hline $\begin{array}{l}\text { Média do período } \\
\text { Period mean }\end{array}$ & & 10,7 & 1,39 & 7,4 & 1,01 & 10,0 & 1,40 & $*$ & $* *$ \\
\hline
\end{tabular}

A, B Médias, na coluna, seguidas por letras maiúsculas distintas, diferem $(P<0,10)$ pelo teste Tukey.

$A, B$ Means, in a column, followed by different capital letters, differ $(P<.10)$ by Tukey test.

* $y=18,222( \pm 3,28)-0,141( \pm 0,05) \mathrm{DIA}^{\#}+0,000463( \pm 0,0002) \mathrm{DIA} \mathrm{A}^{2} \mathrm{CV}=25,9 \% ; R^{2}=0,07 ; P=0,0238$.

** $\quad y=2,341( \pm 0,47)-0,0182( \pm 0,01) D I A+0,000063( \pm 0,00003) D I A^{2} ; C V=27,3 \% ; R^{2}=0,04 ; P=0,0323$.

\# Dias pós-parto (Postpartum days).

R. Bras. Zootec., v.33, n.6, p.1822-1832, 2004 (Supl. 1) 
maiores para o desempenho dos bezerros $(58,2 \%)$ (715 contra $452 \mathrm{~g} /$ dia) (Restle et al., 2004) que para a produção de leite das vacas $(20,6 \%)(4,80$ contra 3,98 L/dia) (Restle et al., 2003).

Não houve efeito do grupo genético da vaca na EFLIT $(\mathrm{P}=0,5664)$ e EFMS $(\mathrm{P}=0,4088)$. Estudando a produção e composição do leite e os ganhos de bezerros Angus, Hereford e Charolês, Melton et al. (1967) relataram 5,$7 ; 4,7$; e $5,2 \mathrm{~kg}$ de leite para cada $\mathrm{kg}$ de ganho de peso, respectivamente, até a desmama aos 175 dias. Alencar (1989) verificou maior eficiência para bezerros Nelore $(5,4)$ em relação aos Canchim $(7,5)$, e Alencar et al. (1996) verificaram valores de 6,58 para bezerros Canchim, 4,81 para os $1 / 2$ Canchim $1 / 2$ Nelore e 6,22 para os bezerros $21 / 32$ Charolês $11 / 32$ Nelore em 231 dias de avaliação, concluindo que a produção de leite da vaca apresenta relação inversa com a eficiência na sua utilização pelo bezerro.

Foi verificado efeito do período pós-parto na EFLIT $(\mathrm{P}=0,0208)$ e na EFMS $(\mathrm{P}=0,0415)$, sendo a equação de regressão quadrática a que melhor se ajustou aos dados. O menor valor de EFLIT $(7,5 \mathrm{~L} / \mathrm{kg})$ foi estimado aos 152 dias, enquanto para a EFMS o ponto de mínima estimado pela equação de regressão foi verificado aos 144 dias com 1,03. Estes resultados são explicados pela variação quadrática do ganho de peso dos bezerros ao longo do período pós-parto, com ponto de máxima ( $688 \mathrm{~g} / \mathrm{dia})$ aos 100 dias (Restle et al., 2004), e pela queda mais acentuada na produção de leite das vacas a partir dos 98 dias de lactação (Restle et al., 2003).

Existem diferenças entre os resultados de várias pesquisas sobre a eficiência de conversão de leite em ganho de peso dos bezerros, em decorrência dos métodos empregados para determinar a produção de leite e de fatores como o período de lactação, se o leite era a única fonte de nutriente do bezerro, o nível nutricional da vaca, a constituição do leite, e do grupo genético da vaca e do bezerro. Contudo, diversos trabalhos relatam comportamento linear decrescente com o avanço do período pós-parto. Trabalhando com animais Aberdeen Angus, Drewry et al. (1959) estimaram que o requerimento de leite necessário para produzir cada $\mathrm{kg}$ de ganho foi 12,5 ; 10,8; e 6,3 no primeiro, terceiro e sexto mês de lactação, respectivamente.

Estudando os efeitos da produção de leite sobre o desenvolvimento de bezerros da raça Canchim, Alencar (1987) verificou que foram necessários 10,3; 8,$2 ; 3,6$ e $8,0 \mathrm{~kg}$ de leite para produzir $1,0 \mathrm{~kg}$ de ganho de peso do nascimento aos $30 ; 120$ e 240 dias e dos
30 aos 120 dias de idade, respectivamente. Robison et al. (1978) e Leal \& Freitas (1982) explicam que isto ocorre porque a correlação entre a produção de leite das vacas e o ganho de peso dos bezerros diminui com o avanço do período de lactação, em razão de a taxa de ganho de peso depender mais da ingestão de forragem que da ingestão de leite (Boogs et al., 1980; Holloway et al., 1982). No presente estudo, este decréscimo na eficiência não foi tão significativo, passando de 10,7 para $8,4 \mathrm{~L} / \mathrm{kg}$ para EFLIT e de 1,39 para $1,21 \mathrm{~kg} \mathrm{MS} / \mathrm{kg}$ para EFMS, respectivamente, do primeiro para o último período de avaliação, provavelmente em decorrência das correlações significativas verificadas, tanto para peso quanto para ganho de peso diário, mesmo nos períodos mais avançados da lactação (Restle et al., 2004).

Na Tabela 2, estão apresentados os valores médios referentes aos requerimentos diário e total de nutrientes digestíveis totais (NDT), em kg, para mantença e produção de leite das vacas. O requerimento diário de NDT não apresentou diferença $(\mathrm{P}=0,2827)$ entre os grupos genéticos das vacas, sendo as médias verificadas de 4,9 kg/dia para as $\mathrm{CH}$ e 4,8 kg/dia para as NE. Quando se quantificou o total de NDT requerido pelas vacas do parto ao desmame dos bezerros, novamente o comportamento entre os grupos genéticos foi similar ( $\mathrm{P}=0,2590)$, com valores médios de 1069,0 $\mathrm{kg}$ para vacas $\mathrm{CH}$ e $1038,1 \mathrm{~kg}$ para as NE. Isto porque a produção de leite das vacas não diferiu entre os grupos genéticos (Restle et al., 2003) durante o período de lactação.

No presente estudo, as correlações indicaram elevada relação entre a produção de leite e os requerimentos de NDT ( $\mathrm{r}=0,57 ; \mathrm{P}=0,0001)$. Tomando como base o requerimento de NDT de manutenção pelo peso da vaca ao parto mais o de produção de leite total até o desmame (235 dias), Ribeiro et al. (2001) verificaram maior requerimento de NDT total para vacas $\mathrm{CH}(913,3 \mathrm{~kg})$ em relação às vacas Aberdeen Angus $(707,9 \mathrm{~kg}$ ), sendo que vacas $\mathrm{CH}$ produziram $13,8 \%$ mais leite por dia que as Aberdeen Angus. Vários estudos relatam a relação positiva entre a produção de leite e o consumo de energia da vaca (Euclides Filho et al., 1983; Ferrel \& Jenkins et al., 1984; Montaño Bermudez et al., 1990; Freeking \& Marshall, 1992), em razão dos maiores requerimentos de mantença ou lactação.

Houve interação significativa entre tipo de pastagem e período pós-parto $(\mathrm{P}=0,0272)$ para exigência diária de NDT. Em ambos os tipos de pastagens

R. Bras. Zootec., v.33, n.6, p.1822-1832, 2004 (Supl. 1) 
Tabela 2 - Médias para exigência diária (NDT/dia) e total (NDT/total) de nutrientes digestíveis totais (mantença + produção de leite) das vacas, em $\mathrm{kg}$, de acordo com a pastagem, o grupo genético e o período pós-parto

Table 2 - Means for daily (NDT/dia) and total (NDT/total) requeriment of total digestible nutrients (maintenance plus milk production) of cows, in $\mathrm{kg}$, according to pasture, cow genetic group and postpartum period

\begin{tabular}{|c|c|c|c|c|c|c|}
\hline \multirow[t]{2}{*}{$\begin{array}{l}\text { Pastagem } \\
\text { Pasture }\end{array}$} & \multirow{2}{*}{$\begin{array}{l}\text { Grupo genético } \\
\text { da vaca } \\
\text { Cow genetic group }\end{array}$} & \multicolumn{3}{|c|}{$\begin{array}{l}\text { Período pós-parto (dias) } \\
\text { Postpartum period (days) }\end{array}$} & \multirow[t]{2}{*}{$\begin{array}{l}\text { Média NDT/dia } \\
\text { Mean TDN/day }\end{array}$} & \multirow{2}{*}{$\begin{array}{l}\text { NDT/total } \\
\text { 0-desm. } \\
\text { 0-weaning }\end{array}$} \\
\hline & & $\begin{array}{c}0-70 \\
\text { NDT/dia }\end{array}$ & $\begin{array}{c}\text { 71-154 } \\
\text { NDT/dia }\end{array}$ & $\begin{array}{l}\text { 155-desm. } \\
\text { NDT/dia } \\
\text { 155-weaning }\end{array}$ & & \\
\hline \multirow{3}{*}{$\begin{array}{l}\text { Cultivada } \\
\text { Cultivated }\end{array}$} & Charolês & 5,4 & 5,3 & 4,6 & 5,1 & 1112,3 \\
\hline & $\begin{array}{l}\text { Charolais } \\
\text { Nelore } \\
\text { Nellore }\end{array}$ & 5,0 & 5,1 & 4,5 & 4,9 & 1074,0 \\
\hline & Média & 5,2 & 5,2 & 4,6 & $*$ & $1093,1 \mathrm{~A}$ \\
\hline \multirow{2}{*}{$\begin{array}{l}\text { Mean } \\
\text { Nativa } \\
\text { Native }\end{array}$} & $\begin{array}{l}\text { Nelore } \\
\text { Nellore }\end{array}$ & 5,3 & 4,8 & 4,2 & 4,7 & 1002,3 \\
\hline & $\begin{array}{l}\text { Média } \\
\text { Mean }\end{array}$ & 5,3 & 4,7 & 4,2 & $* *$ & $1014,0 \mathrm{~B}$ \\
\hline
\end{tabular}

A, B Médias, na coluna, seguidas por letras maiúsculas distintas, diferem $(P<0,10)$ pelo teste Tukey.

$A, B$ Means, in a column, followed by different capital letters, differ $(P<.10)$ by Tukey test.

* $\quad y=5,661( \pm 0,20)-0,004( \pm 0,001) D I A^{\#} ; C V=4,8 \% ; R^{2}=0,50 ; P=0,0001$

** $\quad y=5,673( \pm 0,17)-0,007( \pm 0,001) D I A ; C V=6,9 \% ; R^{2}=0,11 ; P=0,0020$.

\# Dias pós-parto (Postpartum days).

foram verificadas reduções lineares ao longo do período pós-parto, acompanhando o decréscimo verificado na produção de leite das vacas (Restle et al., 2003). Para as vacas mantidas em $\mathrm{PN}$, a redução foi maior em relação àquelas mantidas em $\operatorname{PC}(0,007$ contra $0,004 \mathrm{~kg} \mathrm{NDT} /$ dia), visto que a produção de leite destas últimas apresentou maior persistência (comportamento quadrático) (Restle et al., 2003). Entretanto, quando se quantificou o requerimento total (parto ao desmame) de NDT (NDT/total) das vacas, houve superioridade quando mantidas em PC, em decorrência do acréscimo na produção de leite (Restle et al., 2003), posto que a estimativa desta característica também teve como base este parâmetro. Ao avaliarem o efeito de diferentes sistemas de alimentação na eficiência produtiva de vacas de corte, Ribeiro et al. (2001) verificaram menor exigência de NDT e menor produção diária de leite para vacas mantidas exclusivamente em pastagem nativa em relação às que foram manejadas em pastagem cultivada.

Entre as classes de idade da vaca, verificou-se diferença $(\mathrm{P}=0,0001)$ para NDT/dia e para requerimento total de NDT do parto ao desmame $(\mathrm{P}=0,0004)$, de modo que vacas jovens foram mais exigentes
$(2,3 \mathrm{~kg} / \mathrm{dia}$ e $1162,2 \mathrm{~kg})$ que vacas adultas $(2,2 \mathrm{~kg} / \mathrm{dia}$ e $1040,0 \mathrm{~kg}$ ) e estas mais que as vacas velhas $(2,1 \mathrm{~kg} / \mathrm{dia}$ e $958,6 \mathrm{~kg})$, certamente porque, conforme preconizado pelo NRC (1973), vacas jovens ainda estão em fase de crescimento, necessitando de maior aporte energético.

$\mathrm{Na}$ Tabela 3, encontram-se os valores médios para a quantidade diária de NDT (mantença + produção de leite) requerido pela vaca $(\mathrm{kg})$ para produzir cada kg de ganho de peso do bezerro (EFNDT) e o requerimento total de NDT da vaca (parto ao desmame) para cada $\mathrm{kg}$ de bezerro desmamado (EFDESM), de acordo com o tipo de pastagem, o grupo genético da vaca e o período pós-parto.

Analisando o efeito do tipo de pastagem, verificou-se que quando as vacas foram mantidas em PC, houve maior eficiência $(\mathrm{P}=0,0564)$ para $\operatorname{EFNDT}(7,9)$ em relação às vacas mantidas em $\mathrm{PN}(12,5)$, em torno de $36,8 \%$, diferença confirmada quando se avaliou a EFDESM (5,8 contra 8,2), representando 29,3\%. Apesar de a exigência diária de NDT ter sido superior para as vacas mantidas em PC (Tabela 2), os resultados para EFNDT são explicados pela elevada diferença percentual $(58,2 \%)$ no ganho de peso médio diário dos bezerros mantidos em PC em relação aos que

R. Bras. Zootec., v.33, n.6, p.1822-1832, 2004 (Supl. 1) 
Tabela 3 - Médias para exigências de nutrientes digestíveis totais (mantença + produção de leite) (NDT) da vaca (kg) por $\mathrm{kg}$ de ganho de peso do bezerro (EFNDT) e exigência total de NDT pela vaca por cada kg de bezerro desmamado (EFDESM), de acordo com a pastagem, o grupo genético e o período pós-parto

Table 3 - Means for total digestible nutrients (mantainance and milk production) (NDT) requeriment of cow, in kg, necessary for each $\mathrm{kg}$ of calf weight gain (EFNDT) and total NDT requeriment of cow per each $\mathrm{kg}$ of calf weaned (EFDESM), according to pasture, cow genetic group and postpartum period

\begin{tabular}{|c|c|c|c|c|c|c|}
\hline \multirow[t]{2}{*}{$\begin{array}{l}\text { Pastagem } \\
\text { Pasture }\end{array}$} & \multirow[t]{2}{*}{$\begin{array}{c}\text { Grupo genético da vaca } \\
\text { Cow genetic group }\end{array}$} & \multicolumn{3}{|c|}{$\begin{array}{l}\text { Período pós-parto (dias) } \\
\text { Postpartum period (days) }\end{array}$} & \multirow[t]{2}{*}{$\begin{array}{c}\text { Média EFNDT } \\
\text { Mean EFNDT }\end{array}$} & \multirow[t]{2}{*}{$\begin{array}{l}\text { EFDESM 0-desm. } \\
\text { EFDESM 0-weaning }\end{array}$} \\
\hline & & $\begin{array}{c}0-70 \\
\text { EFNDT }\end{array}$ & $\begin{array}{r}71-154 \\
\text { EFNDT }\end{array}$ & $\begin{array}{l}\text { 155-desm. } \\
\text { EFNDT }\end{array}$ & & \\
\hline \multirow{3}{*}{$\begin{array}{l}\text { Cultivada } \\
\text { Cultivated }\end{array}$} & Charolês & 6,9 & 5,4 & 11,6 & 7,9 & 5,5 \\
\hline & $\begin{array}{l}\text { Charolais } \\
\text { Nelore } \\
\text { Nellore }\end{array}$ & 7,9 & 6,2 & 9,7 & 7,9 & 6,2 \\
\hline & $\begin{array}{l}\text { Média } \\
\text { Mean }\end{array}$ & 7,4 & 5,8 & 10,7 & 7,9 B & $5,8 \mathrm{~B}$ \\
\hline \multirow{3}{*}{$\begin{array}{l}\text { Nativa } \\
\text { Native }\end{array}$} & Charolês & 10,4 & 10,1 & 21,6 & 14,0 & 8,0 \\
\hline & $\begin{array}{l}\text { Charolais } \\
\text { Nelore } \\
\text { Nellore }\end{array}$ & 10,1 & 8,8 & 14,1 & 11,0 & 8,4 \\
\hline & $\begin{array}{l}\text { Média } \\
\text { Mean }\end{array}$ & 10,3 & 9,5 & 17,8 & $12,5 \mathrm{~A}$ & $8,2 \mathrm{~A}$ \\
\hline \multicolumn{2}{|c|}{$\begin{array}{l}\text { Média do período } \\
\text { Period mean }\end{array}$} & 8,8 & 7,6 & 14,2 & $*$ & \\
\hline
\end{tabular}

A, B Médias, na coluna, seguidas por letras maiúsculas distintas, diferem $(P<0,10)$ pelo teste Tukey.

$A, B$ Means, in a column, followed by different capital letters, differ $(P<.10)$ by Tukey test.

* $y=17,032( \pm 4,29)-0,172( \pm 0,07) D^{\prime} A^{\#}+0,0007( \pm 0,00024) D^{2} A^{2} ; C V=27,1 \% ; R^{2}=0,12 ; P=0,0008$

\# Dias pós-parto (Postpartum days).

permaneceram em PN (715 contra $452 \mathrm{~g} / \mathrm{dia}$ ) (Restle et al., 2004). Esta conclusão foi confirmada pela correlação negativa verificada entre o ganho de peso do bezerro e a EFNDT ( $\mathrm{r}=-0,32 ; \mathrm{P}=0,0001)$. Ribeiro et al. (2001) verificaram que as vacas que receberam pastagem cultivada por maior período foram as mais eficientes, ao contrário das que permaneceram apenas em pastagem nativa.

O grupo genético da vaca não influenciou a EFNDT $(\mathrm{P}=0,2823)$ e a $\operatorname{EFDESM}(\mathrm{P}=0,1699)$. Os valores verificados para as vacas $\mathrm{CH}$ e $\mathrm{NE}$ foram de 11,0 e 9,5 para EFNDT e, para EFDESM, de 6,8 e 7,3. Avaliando a relação entre o requerimento de NDT para manutenção e produção de leite do parto ao desmame com o peso à desmama do bezerro, Ribeiro et al. (2001) verificaram que vacas mais leves foram mais eficientes, corroborando os resultados de Euclides Filho et al. (1983), que reportaram melhor eficiência para vacas mais leves e de menor potencial para produção de leite quando estudaram a relação ao consumo total de NDT da vaca mais bezerro pelo peso do bezerro à desmama. Contudo, alguns autores reportam que o grupo genético não representa grande importância na eficiência energética de vacas
(Holloway et al., 1975; Davis et al., 1983).

Similaridade para a EFNDT $(\mathrm{P}=0,1310)$ foi verificada entre as classes de idade da vaca $(11,3$ para vacas jovens, 8,3 para as adultas e 11,0 para as velhas), porém, quando se avaliou a EFDESM, vacas jovens necessitaram 38,7\% mais NDT $(\mathrm{P}=0,0001)$ para produzirem cada $\mathrm{kg}$ de bezerro desmamado $(8,6)$ que as adultas $(6,2)$ e as velhas $(6,2)$.

Houve efeito $(\mathrm{P}=0,0002)$ do período pós-parto, visto que a evolução da EFNDT apresentou comportamento quadrático, com ponto de mínima (6,5) aos 123 dias. Este resultado foi reflexo do comportamento inverso do ganho de peso médio diário dos bezerros, com ponto de máxima (688 g/dia) aos 100 dias (Restle et al., 2004), uma vez que a exigência diária de NDT reduziu linearmente ao longo do período pós-parto (Tabela 2).

$\mathrm{Na}$ Tabela 4, são apresentados os valores médios para quantidade (em $\mathrm{kg}$ ) de bezerros desmamados por $100 \mathrm{~kg}$ de vaca (EFKG) e por unidade de tamanho metabólico (UTM) da vaca (EFTM), de acordo com o tipo de pastagem, o grupo genético da vaca e o período pós-parto.

Houve similaridade entre os grupos genéticos tanto para EFKG $(\mathrm{P}=0,5419)$ quanto para $\mathrm{EFTM}$

R. Bras. Zootec., v.33, n.6, p.1822-1832, 2004 (Supl. 1) 
$(\mathrm{P}=0,7202)$. Com valores de $25,0 \mathrm{~kg} / 100 \mathrm{~kg}$ e $1,14 \mathrm{~kg} /$ UTM para vacas CH e 25,6 kg/100 kg e 1,13 kg/UTM para vacas NE.

Foi verificada interação significativa entre tipo de pastagem e período pós-parto para EFKG $(\mathrm{P}=0,0056)$ e para $\operatorname{EFTM}(\mathrm{P}=0,0008)$, com equações de regressão apresentando comportamento linear crescente. Conforme demonstrado nas equações, as vacas mantidas em $\mathrm{PC}$ produziram mais $\mathrm{kg}$ de bezerros/dia que as mantidas em PN, tanto para EFKG $(0,201$ contra 0,159$)$ quanto para $\operatorname{EFTM}(0,009$ contra 0,007$)$, variação que, ao longo do período, pode ser explicada pelo aumento linear no peso dos bezerros (Restle et al., 2004) e a estabilização ou perda de peso das vacas. A correlação entre o peso vivo do bezerro e a EFKG foi de $0,26(\mathrm{P}=0,0020)$ e entre a EFTM, de 0,28 ( $\mathrm{P}=0,0008)$. Houve influência positiva da produção de leite da vaca na EFKG $(\mathrm{r}=0,14 ; \mathrm{P}=0,0999)$ e na $\operatorname{EFTM}(r=0,16 ; \mathrm{P}=0,0653)$, indicando que o incremento na produção de leite aumenta a quantidade em $\mathrm{kg}$ de bezerros desmamados. Avaliando a relação entre o peso do bezerro ao desmame com o peso da vaca ao desmame, Ribeiro et al. (2001) verificaram maior produtividade em vacas manejadas em pastagem cultivada de inverno em relação às que permaneceram em PN.
Na Tabela 5, estão apresentados os valores médios para algumas características de eficiência avaliadas segundo o tipo de pastagem, sexo do bezerro e a classe de idade da vaca.

Houve interação significativa entre classe de idade da vaca, sexo do bezerro e tipo de pastagem para EFLIT e EFMS. Verificou-se que bezerros machos $(13,3 \mathrm{~L} / \mathrm{kg})$ e bezerros mantidos em PN $(13,2 \mathrm{~L} / \mathrm{kg})$, filhos de vacas velhas, foram os que necessitaram de maior quantidade de leite para cada $\mathrm{kg}$ de ganho de peso. Comportamento similar foi verificado para a EFMS, sendo de 1,81 e 1,79, citados na mesma ordem.

As características de eficiência avaliadas de acordo com o sistema de acasalamento e o grupo genético do bezerro estão apresentadas na Tabela 6. Não houve diferença para EFLIT $(\mathrm{P}=0,9164)$ e EFMS $(\mathrm{P}=0,8358)$ entre sistema de acasalamento e grupo genético dos bezerros.

Para a quantidade de NDT, quando as vacas amamentavam bezerros $\mathrm{F} 1$, o requerimento foi $4,2 \%$ superior $(\mathrm{P}=0,0194)$ em relação às que amamentavam bezerros puros $(5,0$ contra $4,8 \mathrm{~kg} / \mathrm{dia})$. Porém, os resultados obtidos para a EFNDT demonstraram ser mais favoráveis $(\mathrm{P}=0,1072)$ para as vacas que pro-

Tabela 4 - Médias para $\mathrm{kg}$ de bezerros desmamados por $100 \mathrm{~kg}$ de vaca (EFKG) e por unidade de tamanho metabólico da vaca (EFTM), de acordo com a pastagem, o grupo genético da vaca e o período pós-parto

Table 4 - Means for $\mathrm{kg}$ of calves weaned per $100 \mathrm{~kg}$ of cow (EFKG) and per unit of cow metabolic weight (EFTM), according to pasture, cow genetic group and postpartum period

\begin{tabular}{|c|c|c|c|c|c|c|c|c|c|}
\hline \multirow[t]{3}{*}{$\begin{array}{l}\text { Pastagem } \\
\text { Pasture }\end{array}$} & \multirow{3}{*}{$\begin{array}{c}\text { Grupo genético } \\
\text { da vaca } \\
\text { Cow genetic } \\
\text { group }\end{array}$} & \multicolumn{6}{|c|}{$\begin{array}{l}\text { Período pós-parto (dias) } \\
\text { Postpartum period (days) }\end{array}$} & & \\
\hline & & \multicolumn{2}{|c|}{$0-70$} & \multicolumn{2}{|c|}{$71-154$} & \multicolumn{2}{|c|}{$\begin{array}{l}\text { 155-desmame } \\
\text { 155-weaning }\end{array}$} & \multicolumn{2}{|c|}{$\begin{array}{c}\text { Média } \\
\text { Mean }\end{array}$} \\
\hline & & EFKG & EFTM & EFKG & EFTM & EFKG & EFTM & EFKG & EFTM \\
\hline \multirow{4}{*}{$\begin{array}{l}\text { Cultivada } \\
\text { Cultivated }\end{array}$} & Charolês & 12,6 & 0,55 & 28,6 & 1,30 & 41,1 & 1,88 & 27,4 & 1,24 \\
\hline & Charolais & & & & & & & & \\
\hline & Nelore & 11,1 & 0,47 & 27,9 & 1,22 & 41,7 & 1,84 & 26,9 & 1,18 \\
\hline & $\begin{array}{l}\text { Nellore } \\
\text { Média } \\
\text { Mean }\end{array}$ & 11,8 & 0,51 & 28,3 & 1,26 & 41,4 & 1,86 & * & 1 \\
\hline \multirow{4}{*}{$\begin{array}{l}\text { Nativa } \\
\text { Native }\end{array}$} & Charolês & 10,7 & 0,49 & 23,6 & 1,08 & 33,6 & 1,52 & 22,6 & 1,03 \\
\hline & Charolais & & & & & & & & \\
\hline & Nelore & 11,4 & 0,51 & 24,9 & 1,11 & 36,2 & 1,60 & 24,2 & 1,07 \\
\hline & $\begin{array}{l}\text { Nellore } \\
\text { Média } \\
\text { Mean }\end{array}$ & 11,0 & 0,50 & 24,3 & 1,09 & 34,9 & 1,56 & $* *$ & 2 \\
\hline
\end{tabular}

* $y=-2,471( \pm 1,38)+0,201( \pm 0,008) D I A * C V=8,9 \% ; R^{2}=0,89 ; P=0,0001$

** $y=-0,749( \pm 1,07)+0,159( \pm 0,007) D I A ; C V=6,3 \% ; R^{2}=0,93 ; P=0,0001$

$1 y=-0,133( \pm 0,06)+0,009( \pm 0,0004) D I A ; C V=8,4 \% ; R^{2}=0,90 ; P=0,0001$.

$2 y=-0,035( \pm 0,04)+0,007( \pm 0,0003) D I A ; C V=5,8 \% ; R^{2}=0,94 ; P=0,0001$.

\# Dias pós-parto (Postpartum days).

R. Bras. Zootec., v.33, n.6, p.1822-1832, 2004 (Supl. 1) 
Tabela 5 - Médias para litros de leite produzido pela vaca por $\mathrm{kg}$ de ganho peso do bezerro (EFLIT) e kg de matéria seca de leite produzido pela vaca por kg de ganho peso do bezerro (EFMS) de acordo com o sexo do bezerro, pastagem e classe de idade da vaca

Table 5 - Means for litres of milk produced by cows per $\mathrm{kg}$ of calf weight gain (EFLIT) and $\mathrm{kg}$ of dry matter of milk produced by cow per $\mathrm{kg}$ of calf weight gain (EFMS), according to calf sex, pasture and cow age class

\begin{tabular}{|c|c|c|c|c|}
\hline \multirow[t]{2}{*}{$\begin{array}{l}\text { Efeitos } \\
\text { Effects }\end{array}$} & \multicolumn{3}{|c|}{$\begin{array}{c}\text { Classe de idade da vaca } \\
\text { Cow age class }\end{array}$} & \multirow[t]{2}{*}{$\begin{array}{l}\text { Média } \\
\text { Mean }\end{array}$} \\
\hline & $\begin{array}{l}\text { Jovens }(3-4 \text { anos }) \\
\text { Young }(3-4 \text { years old })\end{array}$ & $\begin{array}{l}\text { Adultas }(5-7 \text { anos }) \\
\text { Adult }(5-7 \text { years old })\end{array}$ & $\begin{array}{l}\text { Velhas }(8 \text { ou }+ \text { anos }) \\
\text { Old }(8 \text { or }+ \text { years old })\end{array}$ & \\
\hline
\end{tabular}

\begin{tabular}{|c|c|c|c|c|}
\hline $\begin{array}{l}\text { Sexo bezerro } \\
\text { Calf sex }\end{array}$ & .. & CIT, L/ & & \\
\hline $\begin{array}{l}\text { Macho } \\
\text { Male }\end{array}$ & $9,5 b$ & $7,7 \mathrm{~b}$ & $13,3 \mathrm{a}$ & 10,2 \\
\hline $\begin{array}{l}\text { Fêmea } \\
\text { Female }\end{array}$ & $10,3 a b$ & $9,1 b$ & $6,3 b$ & 8,6 \\
\hline
\end{tabular}

\begin{tabular}{|c|c|c|c|c|}
\hline $\begin{array}{l}\text { Pastagem } \\
\text { Pasture }\end{array}$ & \multicolumn{3}{|c|}{ 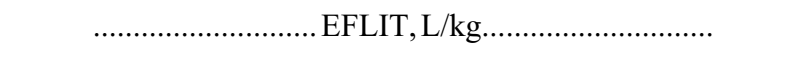 } & \multirow[b]{2}{*}{7,7} \\
\hline $\begin{array}{l}\text { Cultivada } \\
\text { Cultivated }\end{array}$ & $8,5 \mathrm{~b}$ & $8,3 b$ & $6,4 b$ & \\
\hline $\begin{array}{l}\text { Nativa } \\
\text { Native } \\
\text { Média } \\
\text { Mean }\end{array}$ & $11,3 \mathrm{ab}$ & $8,5 \mathrm{~b}$ & $13,2 \mathrm{a}$ & 11,0 \\
\hline $\begin{array}{l}\text { Sexo bezerro } \\
\text { Calf sex }\end{array}$ & \multicolumn{3}{|c|}{$. . \mathrm{EFMS}, \mathrm{kg} \mathrm{MS} / \mathrm{kg} \ldots \ldots \ldots \ldots \ldots \ldots \ldots \ldots \ldots$} & \\
\hline $\begin{array}{l}\text { Macho } \\
\text { Male } \\
\text { Fêmea } \\
\text { Female }\end{array}$ & $\begin{array}{l}1,28 \mathrm{~b} \\
1,42 \mathrm{ab}\end{array}$ & $1,03 b$ & $\begin{array}{l}1,81 \mathrm{a} \\
0,84 \mathrm{~b}\end{array}$ & 1,37 \\
\hline \multicolumn{5}{|l|}{$\begin{array}{l}\text { Pastagem } \\
\text { Pasture }\end{array}$} \\
\hline $\begin{array}{l}\text { Cultivada } \\
\text { Cultivated }\end{array}$ & $1,19 b$ & $1,13 b$ & $0,87 \mathrm{~b}$ & 1,06 \\
\hline $\begin{array}{l}\text { Nativa } \\
\text { Native }\end{array}$ & $1,51 \mathrm{ab}$ & $1,12 b$ & $1,79 \mathrm{a}$ & 1,47 \\
\hline $\begin{array}{l}\text { Média } \\
\text { Mean }\end{array}$ & 1,35 & 1,13 & 1,33 & \\
\hline
\end{tabular}

a, b Médias para o mesmo efeito, seguidas por letras minúsculas distitnas, diferem $(P<0,10)$ pelo teste Tukey.

$a, b$ Means for the same effect, followed by different small letters differ $(P<.10)$ by Tukey test.

duziram bezerros mestiços $(9,4$ contra 11,0), representando diferença de $14,5 \%$. Isto porque bezerros mestiços foram $11,8 \%$ mais pesados ao desmame que bezerros puros (169,1 contra 151,3 kg) (Restle et al., 2004).

Avaliando os grupos genéticos quanto à EFKG, para os bezerros $\mathrm{CH}$ e NE, a produtividade foi similar $(24,5$ contra $22,5 \mathrm{~kg} / 100 \mathrm{~kg})$. Contudo, vacas NE amamentando bezerros filhos de touros $\mathrm{CH}$ foram mais produtivas em relação às vacas $\mathrm{CH}$ amamentando bezerros filhos de touros NE (28,5 contra 25,6 $\mathrm{kg} / 100 \mathrm{~kg}$ ). Na média entre os sistemas de acasalamento, vacas que produziram bezerros mestiços foram mais eficientes que as que produziram bezerros puros $(27,1$ contra $23,5 \mathrm{~kg} / 100 \mathrm{~kg})$, representando diferença de 15,3\%. Resultados favoráveis para produtividade de bezerros/vaca quando as vacas amamentavam bezerros cruzas F1 (Bos taurus $x$ Bos indicus) em relação às vacas que amamentaram bezerros puros (12,1\%) foram reportados por Koger (1976).

Para a EFTM, houve maior produção quando as vacas amamentaram bezerros puros $\mathrm{CH}(1,11 \mathrm{~kg} /$ unidade de tamanho metabólico (UTM) em relação às que amamentaram bezerros puros NE $(0,99 \mathrm{~kg} / \mathrm{UTM})$.

R. Bras. Zootec., v.33, n.6, p.1822-1832, 2004 (Supl. 1) 
Tabela 6 - Médias para litros de leite por $\mathrm{kg}$ de ganho peso do bezerro (EFLIT), kg de MS de leite produzido pela vaca por $\mathrm{kg}$ de ganho peso do bezerro (EFMS), exigência diária $(\mathrm{kg})$ de nutrientes digestíveis totais (mantença + produção de leite) da vaca (NDT), NDT por kg de ganho de peso do bezerro (EFNDT), kg de bezerros produzidos por $100 \mathrm{~kg}$ de vaca (EFKG) e $\mathrm{kg}$ de bezerros produzidos por unidade de tamanho metabólico de vaca (EFTM), de acordo com o sistema de acasalamento e grupo genético dos bezerros

Table 6 - Means for litres of milk produced by cow per $\mathrm{kg}$ of calf weight gain (EFLIT), $\mathrm{kg}$ DM of milk produced by cows per $\mathrm{kg}$ of calf weight gain (EFMS), daily total digestible nutrients (mantainance plus milk production) requeriment of cow (NDT), NDT per $\mathrm{kg}$ of calf weight gain (EFNDT), $\mathrm{kg}$ of calves produced per $100 \mathrm{~kg}$ of cow (EFKG) and $\mathrm{kg}$ of calves produced per unit of cow metabolic weight (EFTM), according to breeding system and calves genetic group

\begin{tabular}{|c|c|c|c|c|c|c|}
\hline \multirow[t]{2}{*}{$\begin{array}{l}\text { Sistema de acasalamento } \\
\text { Breeding system }\end{array}$} & \multicolumn{6}{|c|}{$\begin{array}{c}\text { Características de eficiência } \\
\text { Efficiency characteristics }\end{array}$} \\
\hline & EFLIT & EFMS & NDT & EFNDT & EFKG & EFTM \\
\hline \multicolumn{7}{|l|}{ Puros } \\
\hline \multicolumn{7}{|l|}{ Straigthbred } \\
\hline $\mathrm{CH}$ & 9,4 & 1,26 & 4,8 & 12,6 & $24,5 \mathrm{a}$ & $1,11 \mathrm{a}$ \\
\hline $\mathrm{NE}$ & 9,7 & 1,34 & 4,7 & 9,5 & $22,5 \mathrm{a}$ & $0,99 \mathrm{~b}$ \\
\hline Média puros & 9,5 & 1,30 & $4,8 \mathrm{~B}$ & 11,0 & $23,5 \mathrm{~B}$ & $1,05 \mathrm{~B}$ \\
\hline \multicolumn{7}{|l|}{$\begin{array}{l}\text { Straigthbred mean } \\
\text { F1 }\end{array}$} \\
\hline $1 / 2 \mathrm{CH} 1 / 2 \mathrm{NE}$ & 9,6 & 1,32 & 4,9 & 9,4 & $28,5 \mathrm{a}$ & $1,26 \mathrm{a}$ \\
\hline $1 / 2 \mathrm{NE} 1 / 2 \mathrm{CH}$ & 8,8 & 1,14 & 5,1 & 9,4 & $25,6 b$ & $1,16 \mathrm{a}$ \\
\hline Média F & 9,2 & 1,23 & $5,0 \mathrm{~A}$ & 9,4 & $27,1 \mathrm{~A}$ & $1,21 \mathrm{~A}$ \\
\hline F1 mean & & & & & & \\
\hline Heterose, \% & 3,3 & $-5,4$ & $4,2 * *$ & $-14,5 * * *$ & $15,3 *$ & $15,2 *$ \\
\hline Heterosis, \% & & & & & & \\
\hline
\end{tabular}

* $\quad \mathrm{P}=0,0001(P=.0001) ;{ }^{* *} \mathrm{P}=0,0194(P=.0194) ;{ }^{* * *} \mathrm{P}=0,1072(P=.1072)$.

a,b Médias, na coluna, seguidas de letras minúsculas distintas, na comparação entre $\mathrm{CH}$ e $\mathrm{NE}$ dentro do sistema de acasalamento de puros e entre $1 / 2 \mathrm{CH} 1 / 2 \mathrm{NE}$ e $1 / 2 \mathrm{NE} 1 / 2 \mathrm{CH}$ dentro do sistema de acasalamento de $\mathrm{F} 1$, são diferentes $(P<0,10)$ pelo teste Tukey.

$A, B$ Médias, na coluna, seguidas por letras maiúsculas distintas, na comparação entre puros e $F 1$, são diferentes $(P<0,10)$ pelo teste Tukey.

a,b Means, in a row, followed by different small letters, for the comparison between $\mathrm{CH}$ and $\mathrm{NE}$ within the straigthbred breeding system and between $1 / 2 \mathrm{CH}$ $1 / 2 \mathrm{NE}$ and $1 / 2 \mathrm{NE} 1 / 2 \mathrm{CH}$ within the $\mathrm{F} 1$ system are different $(P<.10)$ by Tukey test.

$A, B$ Means, in a column, followed by different capital letters, for the comparison between straigthbreds and F1, are different (P<.10) by Tukey test.

Entre os animais mestiços, não se verificou diferença significativa, sendo $1,26 \mathrm{~kg} / \mathrm{UTM}$ para os $1 / 2 \mathrm{CH} 1 / 2 \mathrm{NE}$ e $1,16 \mathrm{~kg} / \mathrm{UTM}$ para os $1 / 2 \mathrm{NE} 1 / 2 \mathrm{CH}$. Comparando os sistemas de acasalamento, houve superioridade na produção de kg de bezerros por unidade de tamanho metabólico da vaca quando estas produziram bezerros mestiços $(1,21$ contra $1,05 \mathrm{~kg} / \mathrm{g})$, em torno de $15,2 \%$.

Estes resultados verificados na EFKG e EFTM foram reflexo da superioridade de $10,2 \%$ no ganho de peso do nascimento à desmama dos bezerros mestiços $(626 \mathrm{~g} / \mathrm{dia})$ em relação aos puros $(568 \mathrm{~g} / \mathrm{dia})$ (Restle et al., 2004).

\section{Conclusões}

O uso de pastagem cultivada do parto ao desmame promoveu aumento na eficiência biológica de vacas Charolês ou Nelore amamentando bezerros puros ou mestiços.

Vacas que produziram bezerros mestiços foram mais produtivas que as que produziram bezerros puros, indicando benefício do uso do cruzamento.

\section{Literatura Citada}

ANUAlPEC - Anuário Estatístico da Pecuária Brasileira. FNP. São Paulo: Camargo Soares, 2002. 362p.

ALENCAR, M.M. Efeitos da produção de leite sobre o desenvolvimento de bezerros Canchim. Revista da Sociedade Brasileira de Zootecnia, v.16, n.1, p.1-13, 1987.

ALENCAR, M.M.; OLIVEIRA, F.T.T.; TAMBASCO, A.J. et al. Desenvolvimento pós-desmama e eficiência reprodutiva pós-parto em gado de corte: Influência da produção de leite. Revista da Sociedade Brasileira de Zootecnia, v.22, n.6, p.1012-1018, 1993.

ALENCAR, M.M.; TULLIO, R.R.; CRUZ, G.M. et al. Produção de leite da vaca e desenvolvimento do bezerro em gado de corte. Revista da Sociedade Brasileira de Zootecnia, v.25, n. 1, p.92-101, 1996.

BARCELLOS, J.O.J.; LOBATO, J.F.P.; FRIES, L.A. Eficiência de vacas primíparas Hereford e cruzas Hereford-Nelore acasaladas no outono/inverno ou na primavera/verão. Revista Brasileira de Zootecnia, v.25, n.3, p.414-427, 1996.

BOGGS, D.L.; SMITH, E.F.; SCHALLES, R.R. et al. Effects of milk and forage intake on calf performance. Journal of Animal Science, v.51, n.3, p.550-553, 1980.

BOND, J.; WILTBANK, J.N. Effect of energy and protein on estrus, conception rate, growth and milk production of beef females. Journal of Animal Science, v.30, n.3, p.438-444, 1970.

R. Bras. Zootec., v.33, n.6, p.1822-1832, 2004 (Supl. 1) 
CRUZ, G.M.; ALENCAR, M.M.; TUlliO, R.R. Produção e composição do leite de vacas da raças Canchim e Nelore. Revista Brasileira de Zootecnia, v.26, n.5, p.887-893, 1997.

DREWRY, K.J.; BROWN, C.J.; HONEA, R.S. Relationships among factors associated with mothering ability in beef cattle. Journal of Animal Science, v.18, p.938-946, 1959.

EUCLIDES FILHO, K.; RESTLE, J.; OLSON, T.A. et al. Measures of efficiency of calf production from cows of different size and milking ability. Florida Beef Cattle Research Report, p.13-17, 1983.

FERRELL, C.L.; JENKINS, T.G. Energy utilization by mature, nonpregnant, nonlactating cows of different types. Journal of Animal Science, v.58, n.1, p.234-243, 1984.

FREKING, B.A.; MARSHALL, D.M. Interrelationships of heifer milk production and other biological traits with production efficiency to weaning. Journal of Animal Science, v.70, p.646-655, 1992.

HOLLOWAY, J.W.; BUTTS, W.T.; WORLEY, T.L. Utilization of forage and milk energy by Angus calves grazing fescue or fescue-legume pastures. Journal of Animal Science, v. 55, n.5, p.1214-1223, 1982.

KOGER, M. Resumen y conclusiones. In: KOGER, M.; CUNHA, T.J.; WARNICK, C. (Eds.) Cruzamientos en ganado vacuno de carne. Montevideo: Editorial Hemisferio Sur, 1976. p.536-552.

KRESS, D.D.; DOORNBOS, D.E.; ANDERSON, D.C. Performance of crosses among Hereford, Angus and Simmental cattle with different levels of Simmental breeding: V. Calf production, milk production and reproduction of three to eight-year-old dams. Journal of Animal Science, v.68, p.1910-1921, 1990

LEAL, T.C.; FREITAS, J.E. Correlação entre produção de leite e ganho de peso de bezerros da raça Charolesa. Anuário Técnico do IPZFO, Porto Alegre, v.9, p.91-101, 1982.

LOBATO, J.F.P.; DERESZ, F.; LEBOUTE, E.M. et al. Pastagens melhoradas e suplementação alimentar no comportamento reprodutivo de vacas de corte primíparas. Revista Brasileira de Zootecnia, v.27, n.1, p.47-53, 1998.

MELTON, A.A.; RIGGS, J.K.; NELSON, L.A. et al. Milk production, composition and calf gains of Angus, Charolais and Hereford cows. Journal of Animal Science, v.26, p.804-809, 1967

MOLLETA, J.L.; RESTLE, J. Desempenho em confinamento de novilhos de diferentes grupos genéticos. Ciência Rural, v.22, n.2, p.227-233, 1992

MONTAÑO-BERMUDEZ, M.; NIELSEN, M.K.; DEUTSCHER, G.H. Energy requeriments for maintenance of crossbred beef cattle with different genetic potential for milk. Journal of Animal Science, v.68, n.8, p.2279-2288, 1990.

MOOJEN, J.L.; RESTLE, J.; MOOJEN, E.L. et al. Efeito da época da desmama e da pastagem no desempenho de vacas e terneiros de corte. 1. Desempenho de terneiros. Ciência Rural, v.24, n.2, p.399-403, 1994.

NATIONAL RESEARCH COUNCIL - NRC. Necesidades nutritivas del ganado vacuno lechero. 1.ed. Buenos Aires Editorial Hemisferio Sur, 1973. 75p.

NEVILLE JR., W.E. The influence of dam's milk production and other factors on 120- and 240-day weight of Hereford calves. Journal of Animal Science, v.21, p.315, 1962.
RESTLE, J.; BRONDANI, I.L.; BERNARDES, R.A.C. O novilho superprecoce. In: RESTLE, J. (Ed). Confinamento, pastagens e suplementação para produção de bovinos de corte. Santa Maria: Imprensa Universitária, 1999b. p.191-214.

RESTlE, J.; PACHECO, P.S.; MOLETTA, J.L. et al. Grupo genético e nível nutricional pós-parto na produção e composição do leite de vacas de corte. Revista Brasileira de Zootecnia, v.32, n.3, p.585-597, 2003a.

RESTLE, J.; PACHECO, P.S.; PASCOAL, L.L. et al. Efeito da pastagem, da produção e composição do leite no desempenho de bezerros de diferentes grupos genéticos. Revista Brasileira de Zootecnia, v.33., n.3, p.691-703, 2004.

RESTLE, J.; POLLI, V.A.; SENNA, D.B. Efeito de grupo genético e heterose sobre a idade e peso à puberdade e sobre o desempenho reprodutivo de novilhas de corte. Pesquisa Agropecuária Brasileira, v.34, n.4, p.701-707, 1999a.

RIBEIRO, E.L.A.; RESTLE, J. Desempenho de terneiros Charolês e Aberdeen Angus puros e seus mestiços com Nelore. Pesquisa Agropecuária Brasileira, v.26, n.8, p.1145-1151, 1991.

RIBEIRO, E.L.A.; RESTLE, J.; ROCHA, M.A. et al. Eficiência produtiva em vacas primíparas das raças Aberdeen Angus e Charolês. Revista Brasileira de Zootecnia, v.30, n.1, p.125-132, 2001.

RIBEIRO, E.L.A.; RESTLE, J.; PIRES, C.C. Produção e composição do leite de vacas Charolês e Aberdeen Angus amamentando bezerros puros ou mestiços. Pesquisa Agropecuária Brasileira, v.26, n.8, p.1267-1273, 1991.

ROBISON, O.W.; YUSUFF, M.K.M.; DILLARD, E.U. Milk production in Hereford cows I. Means and correlations. Journal of Animal Science, v.47, n.1, p.131-135, 1978.

STATISTICAL ANALISYS SYSTEMS - SAS. User's guide: Version 6, v.2. Cary: 1997. 1052p.

SENNA, D.B. Desempenho reprodutivo e produção de leite de vacas de quatro grupos genéticos, desterneiradas precocemente, submetidas a diferentes períodos de pastagem cultivada. Santa Maria: Universidade Federal de Santa Maria, 1996, 85p. Dissertação (Mestrado em Zootecnia) - Universidade Federal de Santa Maria, 1996.

TOTUSEK, R.; ARNETT, D.W.; HOLLAND, G.L. et al. Relation of estimation method, sampling interval and milk composition to milk yield of beef cows and calf gain. Journal of Animal Science, v.37, n.1, p.153-158, 1973.

WYATT, R.D.; GOULD, M.B.W.; TOTUSEK, R. Effect of milk level and biological type on calf growth and performance. Journal of Animal Science, v.45, n.5, p.1138-1145, 1977.

Recebido em: 18/06/03 Aceito em: 30/03/04 